\section{Topical ideas for HIV}

\author{
By Lev Osherovich, Senior Writer
}

Two studies propose the use of known compounds to block HIV at the vaginal epithelium, the most common site of initial infection. The compounds, glycerol monolaurate, a mild surfactant, and griffithsin, an algal protein, showed efficacy in animal and tissue culture models of HIV infection through distinct but complementary mechanisms. The challenge now is to formulate the prophylactic agents.

Glycerol monolaurate (GML) has already been studied in Phase I trials for other indications and is thus ready for industry partnering for an efficacy trial, according to the authors of the Nature paper, who were led by Ashley Haase, professor of microbiology at the University of Minnesota. ${ }^{1}$ Indeed, one member of the research team is already working on applications of GML for sexually transmitted infections with Johnson \& Johnson.

Meanwhile, griffithsin was manufactured and tested by an industry-academic team led by Kenneth Palmer, associate professor of pharmacology and toxicology at the University of Louisville School of Medicine. ${ }^{2}$ Other team members included researchers from Kentucky Bioprocessing LLC, a company the specializes in producing transgenic proteins in plants, and Intrucept Biomedicine LLC, which focuses on the development of antiviral biotherapeutics that block viral entry. The study appeared in the Proceedings of the National Academy of Sciences.

The two companies picked up pieces of tobacco-based protein expression technology from Large Scale Biology Corp., which closed its doors in 2005.

\section{Washing HIV away}

Haase told SciBX that his team discovered the in vivo effects of GML on HIV while studying how the infection spreads from the vaginal epithelium to the $\mathrm{CD} 4^{+} \mathrm{T}$ cells that serve as the main hosts for the virus.

Researchers visualized how simian immunodeficiency virus (SIV) infects macaque monkeys via molecular probes of cervical and vaginal tissue harvested from animals shortly after intravaginal SIV inoculation.

Haase's team discovered that the virus initially infected epithelial cells, triggering an inflammatory response that helped to spread the infection to $\mathrm{CD} 4^{+} \mathrm{T}$ cells.

"Infection starts with only a small patch, but later there's an explosive expansion of infected cells," he said. "We started mapping the clusters of infection and found that new sites of expansion were occurring at sites of inflammation."
The team found that local inflammation prompted the recruitment and activation of dendritic cells (DCs) at sites of infection. DCs are innate immune cells that sponge up viral antigens and present them to $\mathrm{CD}^{+} \mathrm{T}$ cells along with stimulatory cytokines, triggering an acquired immune response. Indeed, Haase's team found that $\mathrm{CD} 4^{+} \mathrm{T}$ cells arrived on the scene shortly after DC activation.

Haase hypothesized that these newly arrived $\mathrm{CD} 4^{+} \mathrm{T}$ cells were the primary culprits behind systemic infection, as viral particles in the vaginal epithelium are unable to spread without the help of these host immune cells (see Figure 1, "Blocking early steps in HIV infection").

"The virus can probably infect individual cells at a number of sites through the vaginal epithelium, but this isn't enough to spread the infection," said Haase. It is the subsequent innate immune response that sets off the truly pathogenic infection, he added.

Haase thus reasoned that blocking vaginal inflammation could prevent systemic infection. To test this idea, he collaborated with

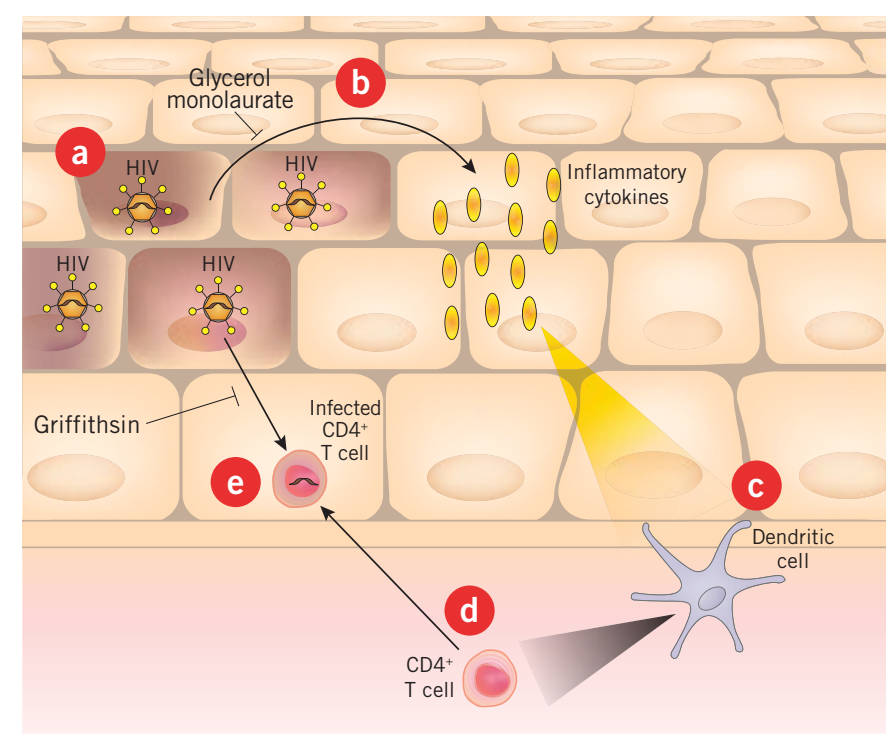

Figure 1. Blocking early steps in HIV infection. Two studies propose ways to block the early stages of HIV infection at the vaginal epithelium based on a mechanism for infection of $\mathrm{CD}^{+} \mathrm{T}$ cells by HIV. As described in Nature, ${ }^{1}$ localized infection of vaginal epithelial cells by HIV [a] leads to production of inflammatory cytokines [b] and recruitment of dendritic cells (DCs) [c]. Activated DCs then recruit $C D 4^{+} T$ cells to the site of infection $[d]$, and the $C D 4^{+} T$ cells then become infected by the virus [e].

The Nature paper proposes that glycerol monolaurate (GML), a mild surfactant, prevents virally induced inflammation and could thus be used to block $\mathrm{CD} 4^{+}$cell recruitment and infection. A report in Proceedings of the National Academy of Sciences suggests that griffithsin, an algae-derived protein, could be used topically in the vaginal epithelium to block HIV infection of $\mathrm{CD} 4^{+} \mathrm{T}$ cells. ${ }^{2}$ 
Patrick Schlievert, professor of microbiology at the University of Minnesota.

The group chose GML because the molecule is a well-studied antagonist of epithelial inflammation and has previously been shown to prevent toxic shock syndrome and microbial infections, according to Schlievert.

"We thought that if we could prevent inflammation we could prevent many different organisms_-including SIV_-from getting through" the innate defense system of the vaginal mucosa to create full-blown infection, he added.

The team found that in cultured human epithelial cells and in the vaginal fluid of monkeys, GML prevented the production of inflammatory cytokines triggered by the virus compared with what was seen in mock-treated controls.

Furthermore, five monkeys treated with a topical solution of GML and shortly thereafter inoculated with SIV had no inflammation and did not develop systemic infections in contrast to mock-treated controls. One of the monkeys subsequently did develop a systemic infection, perhaps because of latent virus that outlasted
"Infection starts with only a small patch, but later there's an explosive expansion of infected cells."

-Ashley Haase,

University of Minnesota bound to HIV particles and inhibited their infectivity compared with what was seen in mock-treated controls.

Although the recombinant protein was somewhat less potent than native griffithsin purified from algae, the recombinant version did block HIV infection in cultured human cervical explants compared with what was seen in untreated controls.

Encouragingly, tobacco-grown griffithsin did not elicit inflammation in cultured human cervical explants or in a rabbit assay of vaginal irritation, relieving concerns about the compound's suitability for use in sensitive vaginal tissue.

In addition to his academic post, Palmer is a cofounder and managing director of Intrucept Biomedicine, a virtual biotech that spun out of the University of Louisville's James Graham Brown Cancer Center. Intrucept partnered to produce griffithsin with Kentucky Bioprocessing, which acquired the patents for the transgenic tobacco technology used in the PNAS study from Large Scale Biology, according to Kentucky Bioprocessing chairman Hugh Haydon.

Gregory Pogue, VP of business development at venture firm Emergent Technologies Inc. and a managing director of Intrucept, said the the initial dose of GML.

Schlievert's team has completed Phase I safety trials of GML for bacterial vaginosis and candidiasis, which he said should pave the way for HIV prevention trials if further animal studies continue to yield good results.

Preclinical studies indicate that GML is also effective against Gramnegative bacteria such as Neisseria gonorrhoeae, which causes gonorrhea. With GML, "the potential is very large to manage all kinds of vaginal infection, not just HIV," said Schlievert.

Haase said his team's next step is to scale up the monkey SIV infection studies to optimize the dose regimen and to confirm that GML produces a similar effect in human vaginal epithelial cultures.

Although Haase said the Nature study led to no new patents, Schlievert has previously filed a patent for the use of GML for Gramnegative infections. Schlievert said his team is working with Johnson \& Johnson to develop GML for sexually transmitted infections.

The pharma company declined to comment on the Nature study.

\section{Protein prophylactic}

Meanwhile, the University of Louisville team reported the large-scale production of griffithsin, an algal protein that binds and kills HIV particles, in transgenic tobacco plants.

Previous studies identified griffithsin as a potent inhibitor of HIV entry. ${ }^{3}$ The molecule works by blocking viral glycoproteins that are needed for HIV to recognize target cells. The problem was that the molecule had not been available in sufficiently large amounts to test in animal models of viral infection.

"People have been proposing protein components in microbicides for quite some time, but the stumbling block has been that the active agent has to be available in large quantities at low cost," said Palmer. "Our study demonstrates that, using the economies of scale in agriculture, it's possible to manufacture griffithsin in bulk."

Palmer's team engineered tobacco plants to produce recombinant griffithsin. In a cell-culture assay of HIV infection, the purified protein next step is to figure out how to formulate griffithsin for topical use.

"We now need to identify a vehicle for the release of griffithsin in the vaginal cavity," said Pogue, who coauthored the PNAS study. "There's not a great deal of work in researching creams and gels for protein release, so this will require some investigation. It's a matter of doing controlled-release studies."

Albert Profy and Zeda Rosenberg think that testing whether griffithsin can prevent SIV in monkeys is a logical next step. Profy is VP of preclinical development at Endo Pharmaceuticals Holdings Inc. and Rosenberg is CEO of the International Partnership for Microbicides Inc. (IPM).

Palmer did caution that "SIV hasn't been very effective in predicting efficacy," and that human trials of griffithsin are now more attractive, thanks to the improved scale of production made possible by transgenic tobacco.

"I would certainly like to work with someone in the SIV model, but people have their doubts about whether it's a good one. I don't see SIV testing as a necessary step along the way" to human trials, Palmer added.

Intrucept and Kentucky Bioprocessing hold a license to griffithsin from the National Cancer Institute, which Pogue said holds composition of matter and use patents on the protein. He added that Intrucept is seeking investors and partners to develop the compound.

\section{The road ahead}

In addition to efficacy, griffithsin and GML will face challenges of formulation, safety and ease of use, according to Rosenberg.

"They're both very early leads in drug development," she said. "They need to generate a much more comprehensive toxicology package. The compounds will need to be formulated for topical use."

Formulations also must be convenient and relatively long-lived to achieve high compliance in trials and in the field, Rosenberg noted.

IPM is a not-for-profit company focused on HIV microbicides 
for women in developing countries. The company's lead products are gel-based and vaginal ring formulations of the non-nucleotide reverse transcriptase inhibitor dapivirine. They have completed Phase I trials.

Thus far, topical antimicrobials for HIV prevention have produced lackluster results. Indeed, Phase II and III trials of nonoxynol-9 being conducted by Columbia Laboratories Inc. were halted in 2000 when it became apparent that the over-the-counter spermicidal and viricidal detergent promoted inflammation and actually increased infection rates. $^{4}$

Earlier this year, PRO 2000, a polyanionic microbicidal gel from Indevus Pharmaceutics Inc. (now part of Endo) missed the primary endpoint in a Phase II/IIb trial to prevent HIV infection in women. Endo expects results from a global Phase III trial sponsored by the U.K.'s Medical Research Council later this year.

Profy said that irrespective of GML's effect on inflammation, the compound may work as a mild detergent that destroys viral particles. If so, GML's proposed mechanism of action may be a red herring and the compound may in fact work similarly to nonoxynol-9.

"GML has some anti-inflammatory effects in vitro, but it's hard to know whether that explains the protection they see in monkeys," said Profy. "They use 5\% GML, and high concentrations of detergents can be viricidal."

Schlievert countered that whereas nonoxynol-9 disrupted cell membranes and thus triggered inflammation, the much milder GML has the opposite effect. He said it stabilizes cell membranes and modulates intracellular signaling to turn down inflammatory cues.

Osherovich, L. SciBX 2(16); doi:10.1038/scibx.2009.645

Published online April 23, 2009

REFERENCES

1. Li, Q. et al. Nature; published online March 5, 2009; doi:10.1038/nature07831

Contact: Ashley Haase, University of Minnesota, Minneapolis, Minn. e-mail: haase001@umn.edu

Contact: Pat Schlievert, same affiliation as above e-mail: schli001@umn.edu

2. O'Keefe, B.R. et al. Proc. Natl. Acad. Sci. USA; published online March 30, 2009; doi:10.1073/pnas.0901506106

Contact: Kenneth Palmer, Owensboro Cancer Research Program, Owensboro, Ky.

e-mail: kepalm02@gwise.louisville.edu

3. Emau, P. et al. J. Med. Primatol. 36, 244-253 (2007)

4. Van Damme, L. et al. Lancet 360, 971-977 (2002)

COMPANIES AND INSTITUTIONS MENTIONED

Columbia Laboratories Inc. (NASDAQ:CBRX), Livingston, N.J.

Emergent Technologies Inc., Austin Texas

Endo Pharmaceuticals Holdings Inc. (NASDAQ:ENDP), Chadds Ford, $\mathrm{Pa}$ International Partnership for Microbicides Inc., Silver Spring, Md. Intrucept Biomedicine LLC, Owensboro, Ky.

James Graham Brown Cancer Center, Louisville, Ky. Johnson \& Johnson (NYSE:JNJ), New Brunswick, N.J. Kentucky Bioprocessing LLC, Owensboro, Ky.

Medical Research Council, London, U.K.

National Cancer Institute, Bethesda, Md.

University of Louisville School of Medicine, Louisville, Ky. University of Minnesota, Minneapolis, Minn. 